\title{
Abnormal Differentiation and Proliferation of Coronary Arterial Endothelium in Hypoplastic Left Heart Syndrome
}

Authors: Zhiyun Yu, BS ${ }^{1,2,3,19}$, Xin Zhou, PhD ${ }^{4,5,19}$, Victor Pastrana-Gomez, BS ${ }^{1,2}$, Lei Tian, $\mathrm{PhD}^{5}$, Timothy J. Nelson, MD, PhD6,7,8,9, Michael P. Snyder, PhD4, Nian Wang, PhD ${ }^{10,11}$, Seema Mital, MD ${ }^{12}$, David Chitayat, MD ${ }^{13,14}$, Joseph C. Wu, MD, PhD ${ }^{5,15,16,17}$, Marlene Rabinovitch, MD5,18, Sean M. Wu, MD, PhD $5,15,16,18$, Yifei Miao, PhD ${ }^{1,2,3,5,18^{*}}$, Mingxia Gu, $\mathrm{MD}, \mathrm{PhD}^{1,2,3,5,18^{*}}$,

\section{Affiliations}

1. Perinatal Institute, Division of Pulmonary Biology, Cincinnati Children's Hospital Medical Center, Cincinnati, $\mathrm{OH}$

2. Center for Stem Cell and Organoid Medicine, CuSTOM, Division of Developmental Biology, Cincinnati Children's Hospital Medical Center, Cincinnati, $\mathrm{OH}$

3. University of Cincinnati School of Medicine, Cincinnati, $\mathrm{OH}$

4. Department of Genetics, Stanford School of Medicine, Stanford, CA

5. Cardiovascular Institute, Stanford School of Medicine, Stanford, CA

6. Division of Pediatric Cardiology, Department of Pediatric and Adolescent Medicine, Mayo Clinic, Rochester, MN

7. Department of Molecular Pharmacology \& Experimental Therapeutics, Mayo Clinic, Rochester, MN

8. General Internal Medicine and Transplant Center, Department of Internal Medicine, Mayo Clinic, Rochester, MN

9. Center for Regenerative Medicine, Mayo Clinic, Rochester, MN

10. Department of Radiology and Imaging Sciences, Indiana University, Indianapolis, IN

11. Stark Neurosciences Research Institute, Indiana University, Indianapolis, IN

12. Department of Pediatrics, Hospital for Sick Children, University of Toronto, Toronto, ON M5G 1X8, Canada

13. Division of Clinical and Metabolic Genetics, Department of Pediatrics, The Hospital for Sick Children, University of Toronto, Toronto, ON M5G 1X8, Canada

14. The Prenatal Diagnosis and Medical Genetics Program, Department of Obstetrics and Gynecology, Mount Sinai Hospital, University of Toronto, Toronto, ON M5G 1X5, Canada

15. Institute for Stem Cell Biology and Regenerative Medicine, Stanford School of Medicine, Stanford, CA

16. Department of Medicine, Division of Cardiovascular Medicine, Stanford School of Medicine, Stanford, CA

17. Department of Radiology, Stanford University School of Medicine, Stanford, CA

18. Department of Pediatrics, Division of Pediatric Cardiology, Stanford School of Medicine, Stanford, CA

19. These authors contributed equally. 


\section{Author for Correspondence}

Yifei Miao, PhD

Cincinnati Children's Hospital Medical Center

Room R4.467; MLC 7029

3333 Burnet Ave

Cincinnati, $\mathrm{OH} 45229$

Phone: 513-636-0337

Email: Yifei.Miao@cchmc.org

Mingxia Gu, MD, PhD

Cincinnati Children's Hospital Medical Center

Room R4.464; MLC 7029

3333 Burnet Ave

Cincinnati, $\mathrm{OH} 45229$

Phone: 513-636-0337

\section{Email: Mingxia.Gu@cchmc.org}

\section{Abstract:}

Hypoplastic left heart syndrome (HLHS) is a severe form of single ventricle congenital heart disease characterized by an underdevelopment of the left ventricle. Early serial postmortem examinations revealed high rate of coronary artery abnormalities in HLHS fetal hearts, which may impact ventricular development and intra-cardiac hemodynamics, leading to a poor prognosis after surgical palliations. Previous study reported that endothelial cells (ECs) lining the coronary vessels showed DNA damage in the left ventricle of human fetal heart with HLHS, indicating that EC dysfunction may contribute to the coronary abnormalities in HLHS.

To investigate the underlying mechanism of HLHS coronary artery abnormalities, we profiled both human fetal heart with an underdeveloped left ventricle (ULV) and ECs differentiated from induced pluripotent stem cells (iPSCs) derived from HLHS patients at single cell resolution. CD144+/NPR3- vascular ECs were selected and further classified as venous EC (NR2F2high), arterial EC (EFNB2high) and late arterial EC (GJA5high) 
subclusters based on previously reported marker genes. To study the arterial phenotype, we specifically generated iPSC-arterial ECs (AECs, CD34+CDH5+CXCR4+NT5E-/low) derived from three HLHS patients and three age-matched healthy controls to further dissect the phenotype of HLHS-AECs.

As compared to normal human heart and control iPSC-ECs respectively, ULV late arterial EC subcluster and HLHS iPSC-EC arterial clusters showed significantly reduced expression of arterial genes GJA5, DLL4, and HEY1. Pathway enrichment analysis based on differentially expressed genes revealed several defects in late AEC cluster from ULV compared to normal human heart, such as impaired endothelial proliferation, development and Notch signaling. HLHS iPSCs exhibited impaired AEC differentiation as evidenced by the significantly reduced CXCR4+NT5E-/low AEC progenitor population. Consistent with human heart transcriptomic data, matured HLHS iPSC-AECs also showed a lower expression of the arterial genes such as GJA5, DLL4, HEY1 which are also downstream of NOTCH signaling. Additionally, matured HLHS iPSC-AECs showed significantly decreased expression of cell proliferation marker Ki67 and G1/S transition genes (CCND1, CCND2) compared with controls. Interestingly, NOTCH ligand Jag1 treatment significantly rescued this cell proliferation defect in HLHS AECs, accompanied by upregulation of various G1/S transition genes.

In summary, we found that coronary AECs from HLHS showed impaired arterial development and proliferation downstream of NOTCH signaling. These functional defects in HLHS coronary AECs could contribute to the vascular structure malformation and impaired ventricular development. 


\section{Main text:}

Hypoplastic left heart syndrome (HLHS) is a severe form of single ventricle congenital heart disease (CHD) characterized by the underdevelopment of the left ventricle, mitral valve, aortic valve, and ascending aorta. Additionally, coronary artery abnormalities such as thickened wall, kinking arteries and ventriculo-coronary arterial connection have been revealed by serial postmortem examinations ${ }^{1}$, which may impact ventricular development and intra-cardiac hemodynamics, leading to a poor prognosis after surgical palliation ${ }^{1}$. However, it is unclear if there is an intrinsic defect in the coronary vessels. A previous study reported that endothelial cells (ECs) lining the coronary vessels showed augmented DNA damage in the left ventricle of HLHS human fetal hearts ${ }^{2}$, suggesting that EC dysfunction may contribute to the coronary malformation in HLHS. By profiling both human fetal heart with an underdeveloped left ventricle (ULV) and generic ECs differentiated from induced pluripotent stem cells (iPSCs) derived from HLHS patients at single cell resolution, we uncovered an abnormal population of coronary arterial ECs with impaired differentiation and proliferation in HLHS.

Firstly, generic heart ECs $\left(\mathrm{CDH}^{+}\right)$, including endocardial $\left(\mathrm{NPR}^{+}\right)$and vascular ECs (NPR3-), were enriched from dissociated the human fetal heart (healthy control vs. ULV) and iPSC derived ECs (iPSC-ECs, healthy control vs. HLHS) ${ }^{3}$. Multiple EC subpopulations were determined by single-cell RNA-seq (scRNA-seq), and vascular ECs (both fetal heart and iPSC-ECs) ${ }^{3}$ were further selected for downstream analysis (A). 
We identified six sub-clusters in fetal heart vascular ECs (B), of which four major clusters (Cluster $0,1,2,3)$ contained the majority of the overlapped control and patient cells and were, therefore, selected for downstream analysis. Based on cell-type-specific markers, they were assigned as vein (NR2F2+, Cluster 0\&2), artery (EFNB2+ $2^{+}$Cluster 1\&3), and late artery $\left(\text { GJA5 } 5^{+} \text {, Cluster } 1\right)^{4}(\mathbf{C})$. iPSC-vascular ECs were heterogenous and classified into four sub-clusters (D), where Clusters 0\&1, although had low expression of venous marker NR2F2, predominantly recapitulated the human fetal arterial clusters (high EFNB2, GJA5) (E), possibly due to the high VEGF in the differentiation medium that induced an arterial cell fate.

We observed a significant reduction in the expression of arterial markers (GJA5, DLL4, HEY1) in arterial ECs (AECs) from both fetal ULV (Cluster 1) and HLHS iPSC-ECs (Cluster 0\&1) as compared to the controls (F). To further understand the intrinsic pathological deficits in HLHS coronary AECs, Gene Ontology (GO) analysis was performed based on the differentially expressed genes (DEGs) between the primary ECs from normal fetal heart and ULV (G). Compared to controls, ULV-vascular ECs showed defects in cell junction, cell migration, and blood vessel morphogenesis pathways (left in G). Interestingly, we found that among four subclusters, the ULV C1 late arterial cluster exhibited the most significant defects in endothelial proliferation, development, Notch signaling (left in $\mathbf{G}$ ), and increased DNA damage and cell cycle arrest (right in $\mathbf{G}$ ).

To further understand the disease phenotypes and underlying mechanisms of HLHS coronary AECs, we generated a pure AEC population from iPSCs through modulation of 
VEGF and Notch pathway ${ }^{5}$. Venous ECs were differentiated in parallel as control. AEC and VEC progenitors $\left(\mathrm{CD} 34^{+}\right)$were sorted, characterized, and further cultured in maturation medium for downstream analysis. Both AEC and VEC differentiation protocols generated ample $\mathrm{CD} 34{ }^{+} \mathrm{CDH} 5{ }^{+} \mathrm{ECs}$, and the high concentration VEGF in AEC protocol efficiently induced CXCR4+NT5E-llow $\mathrm{AEC}^{5}(\mathbf{H})$. In contrast, low VEGF with Notch inhibition facilitated VEC development, as shown by the CXCR4-NT5E ${ }^{+}$ECs $(\mathbf{H})$. Compared to iPSC-VECs, iPSC-AECs showed significantly higher expression of several arterial markers (CXCR4, EFNB2) and lower expression of venous markers (NR2F2, $A P L N R)(\mathbf{I})$.

Next, pure AECs were generated from three HLHS and age-matched control iPSC lines ${ }^{3}$. HLHS iPSCs exhibited impaired AEC differentiation as evidenced by the significantly reduced CXCR4 ${ }^{+} \mathrm{NT5} \mathrm{E}^{- \text {llow }}$ AEC progenitor population $(\mathbf{J})$. In consistent with scRNA-seq analysis (G), mature HLHS AECs had reduced expression of NOTCH and arterial genes (K), such as GJA5, DLL4, HEY1 (F). Additionally, HLHS AECs demonstrated impaired proliferation, as evidenced by the increased cell cycle arrest at G0/G1 (L), the downregulation of Ki67 and G1/S transition genes (CCND1, CCND2), and the upregulation of $\mathrm{G} 1 / \mathrm{S}$ transition inhibitor $C D K N 2 A / P 16(\mathbf{M})$. We further validated this phenotype in HLHS human fetal heart tissues shown by reduced proliferative AECs labeled by Ki67 (N). Intriguingly, the treatment of NOTCH ligand Jag1 improved cell proliferation in HLHS AECs (0), and upregulated G1/S transition genes downstream of the NOTCH pathway (P), indicating that the proliferation defect in HLHS AECs was at least partially mediated by the NOTCH pathway. 
In summary, we revealed that HLHS coronary AECs demonstrated reduced markers for arterial development and cell proliferation downstream of impaired NOTCH signaling. This AEC functional impairment may explain the decreased vessel density and abnormal coronary artery morphology in fetal heart with HLHS ${ }^{1,2}$. These findings provide new molecular insights into the pathogenesis of HLHS and identify NOTCH signaling as a potential therapeutic target.

\section{Footnotes}

Consent for iPSC generation was obtained from both control and patients under approved IRBs: Mayo Clinic: 10-006845; Stanford: IRB 5443. Tissue collection and use in the research were approved by the University of Washington: IRB STUDY00000380. Human tissue sections were obtained under approved IRBs: Hospital for Sick Children IRB 1000011284; Mount Sinai Hospital REB\# 08-0009-E.

\section{Data sharing}

Raw data and complete methods can be made available upon request from the corresponding author. Single-cell RNA sequencing Seurat object have been deposited in the GEO database under accession number GSE138979.

\section{The Correspondence}


Yifei Miao, PhD, Cincinnati Children's Hospital Medical Center, Room R4.464; MLC 7029, 3333 Burnet Ave, Cincinnati, OH 45229

Email:Yifei.Miao@cchmc.org

Mingxia Gu, MD, PhD, Cincinnati Children's Hospital Medical Center, Room R4.467; MLC 7029, 3333 Burnet Ave, Cincinnati, OH 45229

Email: Mingxia.Gu@cchmc.org

\section{Affiliations}

Department of Genetics (X.Z., M.S.), Cardiovascular Institute (X.Z., L.T., S.M.W., J.C.W., M.R., Y.M., M.G.), Department of Pediatrics, Division of Pediatric Cardiology (S.M.W., M.R., Y.M., M.G.), Institute for Stem Cell Biology and Regenerative Medicine \& Department of Medicine, Division of Cardiovascular Medicine (S.M.W., J.C.W.,), Department of Radiology (J.C.W.), Stanford University School of Medicine, Stanford, CA.

Division of Pediatric Cardiology, Department of Pediatric and Adolescent Medicine (T.J.N.), Department of Molecular Pharmacology \& Experimental Therapeutics (T.J.N.), General Internal Medicine and Transplant Center, Department of Internal Medicine (T.J.N.), Center for Regenerative Medicine (T.J.N.), Mayo Clinic, Rochester, MN.

Department of Radiology and Imaging Sciences (N.W.), Stark Neurosciences Research Institute (N.W.), Indiana University, Indianapolis, IN. 
Department of Pediatrics, Hospital for Sick Children (S.M., D.C.), The Prenatal Diagnosis and Medical Genetics Program, Department of Obstetrics and Gynecology, Mount Sinai Hospital (D.C.), University of Toronto, Toronto, Canada

Perinatal Institute, Division of Pulmonary Biology (Z.Y., Y.M., M.G.), Center for Stem Cell and Organoid Medicine, CuSTOM, Division of Developmental Biology (Z.Y., Y.M., M.G.), Cincinnati Children's Hospital Medical Center, Cincinnati, $\mathrm{OH}$. University of Cincinnati School of Medicine (Z.Y., Y.M., M.G.), Cincinnati, OH.

\section{Acknowledgments}

We thank Drs. Qing Liu and Mr. Eric Wei for scRNA-seq sample preparation. We greatly appreciate the administrative help of Ms. Michelle Fox. We also thank Drs. Mark Mercola, Kristy Red-Horse, James Wells, and Kyle Loh for providing intellectual consultant to the project. We also thank ReGen Theranostics, Inc Rochester, MN as the manufacturer for the iPSC lines.

\section{Sources of Funding}

This work was supported by single ventricle gift fund from Stanford University, Todd and Karen Wanek Family Program for Hypoplastic Left Heart Syndrome, NIH 2R24HD000836-52 from the Eunice Kennedy Shriver National Institute of Child Health and Human Development (The University of Washington Birth Defects Research Laboratory). SM is funded by the Heart and Stroke Foundation of Canada Chair and the Ted Rogers Centre for Heart Research. 


\section{Disclosures}

None

\section{References}

1. Cole CR and Eghtesady P. The myocardial and coronary histopathology and pathogenesis of hypoplastic left heart syndrome. Cardiol Young. 2016;26:19-29.

2. Gaber N, Gagliardi M, Patel P, Kinnear C, Zhang C, Chitayat D, Shannon P, Jaeggi E, Tabori U, Keller G and Mital S. Fetal reprogramming and senescence in hypoplastic left heart syndrome and in human pluripotent stem cells during cardiac differentiation. Am J Pathol. 2013;183:720-34.

3. Miao Y, Tian L, Martin M, Paige SL, Galdos FX, Li J, Klein A, Zhang H, Ma N, Wei Y, Stewart M, Lee S, Moonen J-R, Zhang B, Grossfeld P, Mital S, Chitayat D, Wu JC, Rabinovitch M, Nelson TJ, Nie S, Wu SM and Gu M. Intrinsic Endocardial Defects Contribute to Hypoplastic Left Heart Syndrome. Cell Stem Cell. 2020;27:574-589.e8.

4. Su T, Stanley G, Sinha R, D'Amato G, Das S, Rhee S, Chang AH, Poduri A, Raftrey B, Dinh TT, Roper WA, Li G, Quinn KE, Caron KM, Wu S, Miquerol L, Butcher EC, Weissman I, Quake S and Red-Horse K. Single-cell analysis of early progenitor cells that build coronary arteries. Nature. 2018;559:356-362.

5. Gage BK, Liu JC, Innes BT, Macparland SA, McGilvray ID, Bader GD and Keller GM. Generation of Functional Liver Sinusoidal Endothelial Cells from Human Pluripotent Stem-CellDerived Venous Angioblasts. Cell Stem Cell. 2020;27:254-269.e9. 


\section{Figure Legend:}

A. Schematic illustration of the workflow. ECs were either enzymatically dissociated from fetal heart tissue or differentiated from iPSC for further enrichment by magnetic-activated cell sorting (MACS) using CDH5 antibody. Enriched ECs were subject to 10x Genomics 3' V2 version for scRNA-seq. CDH5+/NPR3- (endocardium excluded) vascular ECs were selected for the downstream analysis using the Seurat package.

Normal Fetal heart, $n=1$, gestation day 83, sex unknown; Underdeveloped left heart (ULV, not defined as HLHS because of the early gestational age (day 84$)$ ), $n=1$, male.

iPSC lines information is shown below. MA: Mitral valve atresia; AA: Aortic atresia; MS: Mitral valve stenosis; ERVF: Early right ventricular failure; PBMC: Peripheral blood mononuclear cell.

\begin{tabular}{|c|c|c|c|c|c|}
\hline iPSC Lines & Age & Age of ERVF & Gender & Phenotype & $\begin{array}{c}\text { iPSC Cell } \\
\text { Source }\end{array}$ \\
\hline Control-1 & 1 year & - & Male & Healthy, no heart disease & $\begin{array}{c}\text { Skin } \\
\text { Fibroblast }\end{array}$ \\
\hline Control-2 & 13 years & - & Male & Healthy, no heart disease & $\begin{array}{c}\text { Skin } \\
\text { Fibroblast }\end{array}$ \\
\hline Control-3 & 25 years & - & Male & Healthy, no heart disease & $\begin{array}{c}\text { Skin } \\
\text { Fibroblast }\end{array}$ \\
\hline HLHS-1 & 15 months & 14 months & Male & MA/AA, ERVF & PBMC \\
\hline HLHS-2 & 14 years & 5 years & Female & MA/AA, ERVF & PBMC \\
\hline HLHS-3 & 2 days & 15 months & Male & MS/AA, ERVF & PBMC \\
\hline
\end{tabular}

B-E. UMAP projections and subcluster classification for fetal heart vascular ECs (B-C) and iPSC-vascular ECs (D-E). (B) UMAP projection of Control vs. ULV patient heart vascular ECs. Control $=1835$ cells; Patient $=3742$ cells. $N=1$ in each group. $(C)$ Four clusters of fetal heart vascular ECs were classified based on cell type-specific markers (NR2F2, vein; EFNB2, artery; GJA5, late artery). Cluster 0, 1, 2, 3 were selected based on statistical power > 0.8. (D) UMAP projection of Control vs. HLHS patient iPSC-vascular 
ECs. Control $=657$ cells; Patient $=584$ cells. $\mathrm{N}=1$ in each group. Subclusters were classified based on Seurat package. (E) Expression of arterial and venous marker genes (from C) in UMAP from iPSC-ECs. UMAP was performed on samples using the first 15 axes from the PCA. Clusters were formed using the neighbor-joining method.

F. Violin plots of JGA5, DLL4, and HEY1 gene expressions per cluster in fetal heart vascular ECs and iPSC-vascular ECs based on B and $\mathbf{D}$. The expression levels are normalized via SCT transform to eliminate the variance caused by sequencing depth.

G. GO analysis based on DEGs from fetal heart vascular ECs. The top enriched pathways shown in fetal hearts are selected in the plots. The GO terms with a q-value $<0.05$ are considered significant. The left panel shows the common signals decreased in patient samples, and the right panel shows the common signals increased in patient samples. GO term numbers are indicated following the Y-axis labels. Wilcox rank test was performed on differentially expressed genes (DEGs) based on scRNA-seq dataset. DEGs were generated under Bonferroni correction $p<0.05$ and logE fold change $>1.2$.

H. Flow cytometry analysis of AEC (high VEGF treated, CXCR4 ${ }^{+}$) and VEC (low VEGF, Notch inhibition treated, $\mathrm{NT}^{+} \mathrm{E}^{+}$) progenitors differentiated from iPSCs. The bottom plots are from the $\mathrm{CD} 34^{+} \mathrm{CDH} 5^{+}$gated fraction in top plots.

I. qPCR analysis of arterial and venous markers in iPSC-AEC/VEC progenitors. Differentiated iPSC-AEC/VEC progenitors were MACS sorted for CD $34^{+}$. Values are normalized to TBP. $\mathrm{N}=3$ Technical repeats for each differentiation protocol.

J. Flow cytometry analysis of AEC progenitors generated from control and HLHS iPSCs. Statistical analysis of $\mathrm{CXCR} 4^{+} \mathrm{AEC}$ proportions out of $\mathrm{CD} 34^{+} \mathrm{CDH} 5^{+} \mathrm{EC}$ population from control and HLHS iPSCs are shown on the right. $\mathrm{N}=3$ individuals in each group. 
K. qPCR analysis of arterial markers in mature control and HLHS iPSC-AECs. N=3 individuals in each group.

L. Propidium iodide (PI) staining cell cycle analysis of mature control and HLHS AECs. $\mathrm{PI}$ intensity peak at $50 \mathrm{~K}$ was gated by FACS as G0/G1 phase. Statistical analysis of cell percentage in the G0/G1 phase from control and HLHS iPSC-AECs are shown on the right. $\mathrm{N}=3$ individuals in each group.

M. qPCR analysis of cell cycle markers in mature control and HLHS iPSC-AECs. N=3 individuals in each group.

N. Immunofluorescence staining of cell proliferation marker Ki67 in human fetal heart tissues with DAPI counterstain. CD31 (endothelium) and a-SMA (smooth muscle cells) were stained to represent coronary artery area. Statistical analysis of the number of Ki67 ${ }^{+}$ AECs per coronary arterial EC area was shown on the right. Coronary AEC area were measured in each region of interest $(\mathrm{ROI}) . \mathrm{ROI}$ size $=5000 \times 5000 \mu \mathrm{m}, 10 \mathrm{ROls}$ were chosen in each individual and the average of Ki67+ AECs/coronary AEC area was calculated in each individual. $N=3$ individuals in each group. Scale bar= $20 \mu \mathrm{m}$.

Patient information for human heart tissue sections for staining in $\mathbf{N}$ is shown below.

\begin{tabular}{|c|c|c|}
\hline Study ID & Age & Congenital Heart Diagnosis \\
\hline Control-1 & Postnatal day 1 & Healthy \\
\hline Control-2 & Postnatal day 10 & Healthy \\
\hline Control-3 & Gestational 12 weeks & Healthy \\
\hline HLHS-1 & Gestational 21 weeks & $\begin{array}{c}\text { Hypoplastic left heart syndrome (HLHS)-modifier, } \\
\text { Endocardial fibroelastosis; Hypoplastic left heart } \\
\text { syndrome (HLHS)-modifier, Endocardial } \\
\text { fibroelastosis; (critical aortic stenosis) }\end{array}$ \\
\hline HLHS-2 & Gestational 17 weeks & $\begin{array}{c}\text { Hypoplastic left heart syndrome (HLHS), Aortic } \\
\text { stenosis. Mitral stenosis, IVS }\end{array}$ \\
\hline HLHS-3 & Gestational 21 weeks & $\begin{array}{c}\text { Hypoplastic left heart syndrome (HLHS)-modifier, } \\
\text { Aortic arch hypoplasia; Hypoplastic left heart } \\
\text { syndrome (HLHS)-modifier, Aortic arch hypoplasia }\end{array}$ \\
\hline
\end{tabular}


O. BrdU cell proliferation assay on iPSC-AECs treated with NOTCH ligands (Jag1 or DIl1 coated on the bottom of the culture plate) or untreated. After BrdU incorporation, iPSCAECs were lysed for luminescent quantification at $O D_{450 .} N=$ four technical repeats in each group.

P. Heatmap of gene expression for HLHS iPSC-AECs rescued by NOTCH ligands. Data from Jag1 or Dll1 treated HLHS-AECs were normalized to untreated control. $\mathrm{N}=3$ biological repeats in each group. FC: fold change to untreated group.

Data are presented as mean $+/-95 \%$ confidence interval. Mean \pm SEM, ns, $p>0.05$; ${ }^{*} p<0.05 ;{ }^{* *} p<0.01 ;{ }^{* * *} p<0.001$, based on unpaired two-tailed t-test. 